\title{
More women in Europe and Australia have dental agenesis than their counterparts in North America
}

\author{
What is the prevalence of dental agenesis of permanent teeth?
}

\begin{abstract}
Polder BJ, Van't Hof MA, Van der Linden FPGM, KuijpersJagtman AM. A meta-analysis of the prevalence of dental agenesis of permanent teeth. Commun Dent Oral Epidermiol 2004; 32: 217-226.
\end{abstract}

Data sources Medline and Embase were searched, using the following keywords: "hypodontia", "oligodontia", "anodontia", "agenesis", and "prevalence or incidence". Reference lists from retrieved articles were also examined.

Study selection For inclusion, an article had to satisfy the following criteria: presence of an English abstract; the sample was representative for the underlying general population; the diagnosis of dental agenesis was based on a radiographic examination; the report presented information on the ethnic background; and the report presented prevalence of agenesis except third molars.

Data extraction and synthesis Data from Caucasian populations in North America, Australia and Europe were included in a metaanalysis. For the prevalence in African-American, Chinese and Arab groups, only indications could be reported because of a limited number of studies. Multiple-regression analysis was applied to evaluate the influence of chronological age, sample size, continent and year of publication. Statistical significance was established at $P<0.05$. The prevalence of agenesis per tooth type, affected patients and number of missing teeth per patient was calculated as far as reported in the papers. For the comparison of the prevalence for males and females, the relative risk (RR) was calculated.

Results The search identified 141 articles, of which 31 met the inclusion criteria. Agenesis differs by continent and gender: the prevalence for both sexes was higher in Europe (males 4.6\%; females 6.3\%) and Australia (males 5.5\%; females 7.6\%) than for NorthAmerican Caucasians (males 3.2\%; females 4.6\%). In addition, the prevalence of dental agenesis in females was significantly higher than in males for all three continents, with an RR of 1.37 (95\% Cl, 1.28-1.45). The mandibular second premolar was the most affected tooth, followed by the maxillary lateral incisor and the maxillary second premolar. Unilateral occurrence of dental agenesis is more common than bilateral occurrence. Bilateral agenesis of maxillary lateral incisors is more common, however, than unilateral agenesis. In most patients, dental agenesis involved only one (48\%) or two teeth (35\%).

Conclusions The prevalence of dental agenesis in Europe and Australia is higher than in North America. The overall prevalence of agenesis in the maxilla is comparable with that in the mandible, but a marked difference was found between both jaws regarding tooth type. Absence of one or two permanent teeth is found in the majority of the subjects with dental agenesis.

Address for correspondence: Bart | Polder, Department of Orthodontics and Oral Biology, University Medical Centre Sint Radboud, PO Box 9101, 6500 HB Nijmegen, The Netherlands. E-mail: orthodontics@dent.umcn.nl

\section{Commentary}

This is a comprehensive systematic review and meta-analysis of the prevalence of dental agenesis of permanent teeth. As stated in the Introduction, the reported prevalence of dental agenesis has varied greatly in the literature. This is a good indication of the need for a systematic review in order to summarise existing prevalence data on dental agenesis in population-based studies.

In the Discussion, the analysis of the heterogeneity and bias between studies is done well. These are key considerations in metaanalysis because they provide more insights than the actual numerical calculation of the effects. ${ }^{1}$

As stated in the Discussion, dental agenesis has a multifactorial origin. A careful consideration of this heterogeneity is provided in the article. Ethnic background, sample size considerations, gender, tooth type and dental arch were analysed. As expected, mandibular second bicuspids, upper laterals and maxillary second bicuspids were the teeth most commonly missing.

Dental agenesis does not only represent an individual clinical problem but also a public health problem. Therefore, more and better-planned epidemiological studies about dental agenesis prevalence in most parts of the world are required. Only in European Caucasian (especially Scandinavian countries) and North American populations have there been sufficient studies to give us an idea of their dental agenesis prevalence.

A couple of considerations could have improved this metaanalysis, but the absence does not diminish the meta-analysis in a significant way. Regarding the Materials and methods, a possible limitation was the use of only two databases (Medline and Embase) in the search for available literature. Several other databases should be included to demonstrate that every effort was made to find the available evidence. ${ }^{2,3}$ For example, the authors here did not search the Cochrane database of systematic reviews, a key primary source. ${ }^{4}$ There is also a potential limitation when only Medline and not Pubmed is searched. Although they are both produced by the same company, Medline and Pubmed follow different logic procedures for their searches. This would potentially produce different search hits although significant overlap normally exists. This is clearly dependent on the search terms.

It is also advisable to use Medline-In Process (see www.ovid. $\mathrm{com} / \mathrm{site} /$ products/ovidguide/premdb.htm for further details), which covers the latest publications not completely indexed at the moment of the search. Lilacs (Latin American and Caribbean Health Science Literature database) is also a good search engine to obtain references from Latin-American journals, but language limitation (Spanish or Portuguese) is a disadvantage. ${ }^{5}$ Relevant articles from Lilacs, if available, could help build a more comprehensive picture of the prevalence of dental agenesis worldwide.

Just stating the search terms is not enough to permit the readers to search for new publications that could potentially add new knowledge in this topic between November 2002 (the final search for this report) and today: a table with the exact terms used would have been desirable. Every electronic database has its own set of terms and rules. 
The inclusion and exclusion criteria for the present search seem to be adequate and logical. The whole process of article selection is nicely explained and easy to follow. Additional tables stating the reasons for the abstracts and article exclusion at the different stages would have added greater clarity.

\section{Practice point}

- This information is especially useful for dental public health bodies: although the prevalence of these cases is very low, their cost is significant and their treatment complex.

\section{Carlos Flores-Mir}

Faculty of Medicine and Dentistry Room 4051A, Dentistry/ Pharmacy Center, University of Alberta, Edmonton, Alberta, Canada
1. Egger M, Smith GD, O'Rourke. Rationale, potentials, and promise of systematic reviews. In Systematic Reviews in Health Care, 2nd Edn. Meta-analysis in Context. Edited by Egger M, Smith GD, Altman DG. London: BMJ Books; 2001; pp. 3-19.

2. Egger $M$, Smith GD. Principles and procedures for systematic reviews. In Systematic Reviews in Health Care 2nd Edn. Meta-analysis in Context. Edited by Egger M, Smith GD, Altman DG. London: BMJ Books; 2001; pp. 23-42.

3. Moher D, Pham B, Klassen TP, et al. What contributions do languages other than English make on the results of meta-analyses?. J Clin Epidemiol 2000; 53:964-972.

4. McKibborn A. Finding the evidence. In Users' Guides to the Medical Literature. A Manual for Evidence-based Clinical Practice Edited by Guyatt GH, Rennie D. Chicago: AMA Press; 2002; pp. 48-53.

5. Juni $P$, Holenstein $F$, Sterne J, Bartlett $C$, Egger M. Direction and impact of language bias in meta-analyses of controlled trials: empirical study. Int J Epidemiol 2002; 31:115-123.

Evidence-Based Dentistry (2005) 6, 22-23.

doi:10.1038/sj.ebd.6400312 